\title{
Embarazos Durante el Ciclo de la Biopsia de Endometrio
}

\author{
Drs: Fabio Sánchez*, Gabriel Devia**, Constanza Díaz de Calle***, \\ Guillermo Henao*, Jorge Mario Mejía*
}

\section{INTRODUCGION}

A pesar de que no existe unanimidad acerca de la importancia de la biopsia de endometrio en el estudio de parejas estériles (1-2), la consideramos de gran utilidad, no sólo porque el endometrio es el reflejo del estado endocrino de la paciente (3), sino porque durante el procedimiento y su estudio es posible diagnosticar alteraciones orǵanicas congénitas o adquiridas, procesos agudos o crónicos (4) y además, aunque se ha presentado muy tímidamente, creemos que puede tener algún valor terapéutico. Es así como se ha informado periódicamente un número de embarazos durante el ciclo de la biopsia, fenómeno que se ha atribuído a los cambios mecáni$\cos$ originados en la cavidad uterina o por el contrario a fenómenos neuroendocrinos, iniciados en vagina o en cuello, como sucede en animales inferiores (5-6-7-8-9-10-11). Es nuestro interés informar los embarazos obtenidos en nuestro programa luego de este procedimiento y discutir el posible mecanismo de acción.

\section{MATERIALES Y METODOS}

Se analizaron retrospectivamente las historias clínicas de 223 pacientes estériles que se embaraza-

* Profesor del Grupo de Ginecología Endocrina y Reproducción Humana, Hospital San Vicente de Paúl, Medellín.

** Residente Rotatorio Universidad del Cáuca.

*** Profesora Departamento de Anatomía Patológica, Hospital Universitario San Vicente de Paúl, Medellín. ron en el programa de Ginecología Endocrina y Reproducción Humana, en el Hospital Universitario San Vicente de Paúl, durante un período de cinco años (enero de 1976 a diciembre de 1980) (12).

A todas las parejas se les programó para un estudio integral de todos los factores con el fin de obtener un diagnóstico causal de la esterilidad, el cual se inició con una biopsia de endometrio. La biopsia fue tomada el día 26 del ciclo o dos o tres días antes de la fecha probable de la menstruación. Previa fijación con pinzas y asepsia del cuello uterino, se toma la biopsia de la pared lateral, desde el fondo del útero, con cureta de Novak. Luego del procedimiento las pacientes continuaron en amenorrea. El diagnóstico de embarazo se hizo por evaluación clínica complementada con la prueba inmunológica.

El endometrio se fijó en formol al 10\% y se coloreo con hematoxilina eosina. Todas las biopsias fueron leídas por un mismo patólogo, siguiendo los lineamientos de Noyes y Cols. (3).

\section{RESULTADOS}

De las 223 pacientes embarazadas en el programa de esterilidad, 30 se embarazaron luego de un estímulo vaginal o cervical y se distribuyen así: cinco después del estímulo al cuello (dos luego de prueba post-coito); una por curetaje biopsia fallido; una por cauterización de cuello y una luego de biopsia de cuello) y 25 durante el ciclo post biopsia. Este último grupo de pacientes comprende el $11.1 \%$ 
del total de embarazos y fueron seleccionadas para el presente informe.

Estas pacientes tienen una edad que oscila entre los 20 y 36 años con una media de $27.8 \pm 4.48$ y con uno a nueve años de esterilidad, $2.8 \pm 2.25$ en promedio.

Veintidós de las pacientes quedaron en embarazo durante el ciclo de estudio y sólo tres durante el ciclo de tratamiento (dos con clomifeno y una con dexametasona). Hay un bajo índice de diagnósticos: dos con anovulación crónica y seis con cuerpo lúteo insuficiente. Las 17 pacientes restantes no tuvieron diagnóstico que explicara su esterilidad por lo precoz del embarazo.

Con relación a la lectura del endometrio, se aprecia que en el momento de la biopsia, 11 de las pacientes $(44 \%)$, tenían un endometrio por encima del día 23 del ciclo. El resto de las biopsias, siete, estaban por debajo del día 23; cinco tenían un cuadro sugestivo de maduración asincrónica y dos en fase proliferativa. En cinco de las biopsias se encontró un cuadro sospechoso de endometrio gestacional, uno de ellos con el fenómeno de Arias Stella. (Tabla 1).

\section{TABLA 1}

DISTRIBUCION DE LAS 25 PACIENTES EMBARAZADAS DURANTE EL CICLO DE LA BIOPSIA, DE ACUERDO CON EL INFORME HISTOLOGICO DEL ENDOMETRIO

\begin{tabular}{|l|c|c|}
\hline Histología & Distribución & $\begin{array}{c}\text { Total } \\
\text { de Pacientes }\end{array}$ \\
\hline Secretor & & 18 \\
$17-18$ & 1 & \\
$19-20$ & 3 & \\
$21-22$ & 3 & \\
$23-24$ & 2 & \\
$25-26$ & 8 & 5 \\
$27-28$ & 1 & 2 \\
Mosaico & & \\
Proliferativo & & 25 \\
\hline \multicolumn{2}{|c|}{ Total: }
\end{tabular}

De cinco pacientes no se tienen datos de la terminación del embarazo. Sin embargo, fueron controladas en la consulta prenatal hasta una fecha avanzada; dos hasta 20 semanas, una hasta las 25 y 35 semanas y otra llegó a término pero no se saben los datos del parto. Si se analiza la terminación del embarazo, excluyendo estas 5 pacientes, 14 (70\%) llegaron a término y un $15 \%$ abortos y prematuros. (Tabla 2).

TABLA 2

DISTRIBUCION DE 20 PACIENTES EMBARAZADAS DURANTE EL CICLO DE LA BIOPSIA, DE ACUERDO CON LA FORMA DE TERMINACION DEL EMBARAZO

\begin{tabular}{|c|c|c|}
\hline $\begin{array}{c}\text { Terminación } \\
\text { del Embarazo }\end{array}$ & $\begin{array}{c}\text { No. } \\
\text { de Pacientes }\end{array}$ & Porcentaje \\
\hline Abortos & 3 & $15 \%$ \\
Prematuros & 3 & $15 \%$ \\
A Término & $14 *$ & $70 \%$ \\
\hline Total: & 20 & $100 \%$ \\
\hline
\end{tabular}

* Un embarazo gemelar.

En 5 pacientes no se conoció la forma de terminación del embarazo.

De las 17 pacientes, el parto fue intervenido en seis; cinco de ellas por cesárea $(29.5 \%$ ) y una con Espátulas de Velasco. El resto, (64.7\%) fueron parto normal y espontáneo (Tabla 3). Con relación a los pesos de los niños, cuatro (22\%) estuvieron debajo de los 2.500 gr.; dos de ellos producto de un embarazo gemelar. Hubo mayor frecuencia de niñas (11 de los 18 niños controlados).

TABLA 3

DISTRIBUCION DE 17 EMBARAZOS DURANTE EL CICLO DE LA BIOPSIA, DE ACUERDO CON LA FORMA DE TERMINACION DEL PARTO

\begin{tabular}{|l|c|c|}
\hline $\begin{array}{c}\text { Terminación } \\
\text { del Parto }\end{array}$ & $\begin{array}{c}\text { No. } \\
\text { de Pacientes }\end{array}$ & Porcentaje \\
\hline Normal y & 11 & $64.7 \%$ \\
Espontáneo & 5 & $29.4 \%$ \\
Cesárea & 1 & $5.9 \%$ \\
Espátulas & 17 & $100.0 \%$ \\
\hline Total: & & \\
\hline
\end{tabular}




\section{DISCUSION}

La biopsia de endometrio es un procedimiento útil en el estudio de las pacientes estériles, no sólo porque durante el procedimiento se puede diagnosticar la existencia o no de trastornos anatómicos tales como la atrofia endometrial (13-14), sinequias intracervicales o intrauterinas (15), pólipos (4) y malformaciones congénitas (16), sino porque de su estudio microscópico permite conocer la presencia de procesos infecciosos agudos o crónicos tales como endometritis crónica, granulomatoso tipo TBC (17) y principalmente el influjo hormonal en un momento determinado del ciclo (3).

La frecuencia de abortos en el presente estudio es de un $12 \%$, que es sensiblemente igual al de la población general y al promedio de abortos informados en los embarazos post-biopsia (10). En conclusión, este procedimiento no aumenta la frecuencia de abortos, por lo tanto no se prohiben las relaciones sexuales durante este ciclo como ocurría anteriormente (Cuadro 4).

Esto demuestra que posiblemente el procedimiento, fuera de ser útil para el diagnóstico, es inocuo para el desarrollo del embarazo, siempre y cuando se tome la biopsia en las caras laterales, sitio que es diferente al lugar de mayor implantación del embrión tal como en el fondo y las caras anterior y posterior del útero (8).

Además, la biopsia de endometrio puede tener un efecto benéfico respecto a la implantación ovular bien sea porque produce cambios endocrinos como consecuencia de un reflejo medular originado en vagina o en el cuello que desencadenaría la liberación de gonadotrofinas (9), fenómeno que ocurre en animales inferiores, hipótesis que no fue confirmada por nosotros (18) o bien que el efecto mecánico en el endometrio libera algunas sustancias tales como la anhidrasa carbónica (10) o prostaglandinas (19-20) que produce una mayor reacción decidual y vascularización (9) y como consecuencia una mayor secreción de prolactina, hormona que puede tener algún papel en la implantación ovular (21).

La biopsia es posible que facilite la anidación del óvulo fecundado o mejore su implantación por varios mecanismos. Al liberar un sinnúmero de adherencias intrauterinas, extirpar pólipos o desencade-

TABLA 4

EMBARAZOS DURANTE EL CICLO DE LA BIOPSIA

(RESUMEN DE LA LITERATURA)

\begin{tabular}{|c|c|c|c|c|c|c|c|}
\hline Autor & Año & $\begin{array}{c}\text { No. } \\
\text { Embarazos }\end{array}$ & A Término & Prematuros & Abortos & Ectópicos & $\begin{array}{c}\text { Sin } \\
\text { Datos }\end{array}$ \\
\hline $\begin{array}{l}\text { Mishell } \\
\text { Sturges } \\
\text { Simons } \\
\text { Jackson } \\
\text { Hamblen } \\
\text { Hartman } \\
\text { Wilson } \\
\text { Buxton } \\
\text { Karow } \\
\text { Arronet } \\
\text { Rosenfeld } \\
\text { Sulewski } \\
\text { Sánchez }\end{array}$ & $\begin{array}{l}1940 \\
1940 \\
1946 \\
1947 \\
1957 \\
1961 \\
1966 \\
1969 \\
1971 \\
1973 \\
1975 \\
1980 \\
1983\end{array}$ & $\begin{array}{r}3 \\
10 \\
31 \\
29 \\
10 \\
5 \\
14 \\
22 \\
28 \\
23 \\
18 \\
18 \\
25\end{array}$ & $\begin{array}{r}1 \\
7 \\
31 \\
17 \\
9 \\
4 \\
11 \\
20 \\
22 \\
14 \\
15 \\
13 \\
14\end{array}$ & $\begin{array}{l}3 \\
3\end{array}$ & $\begin{array}{l}2 \\
2 \\
7 \\
1 \\
4 \\
3\end{array}$ & $\begin{array}{l}2 \\
2\end{array}$ & $\begin{array}{l}8 \\
1 \\
3\end{array}$ \\
\hline Total: & & 236 & $\begin{array}{c}178 \\
(75.4 \%)\end{array}$ & $\begin{array}{c}7 \\
(3.0 \%)\end{array}$ & $\begin{array}{c}24 \\
(10.2 \%)\end{array}$ & $\begin{array}{c}5 \\
(2.2 \%)\end{array}$ & $\begin{array}{c}22 \\
(9.2)\end{array}$ \\
\hline
\end{tabular}


nar un proceso inflamatorio que corrija el retardo o el asincronismo del endometrio al acelerar su maduración.

No tenemos una explicacion de los dos embarazos obtenidos en pacientes con endometrio anovulatorio, excepto que la ovulación haya coincidido con el día de la biopsia por un simple azar o que la liberación de prostaglandinas en el endometrio, actúen sobre un folículo preovulatorio y desencadene la ovulación.

Este fenómeno que es fácil de explicar en animales inferiores debido a la circulación útero-ovárica que presentan, no ocurre lo mismo en humanos.

A diferencia de lo que informa Sulewski (11), nueve pacientes con endometrio secretor por encima del día 25 llegaron a termino. El aborto sólo ocurrió en tres casos; una paciente con endometrio anovulatorio, otra con mosaico y otra con secretor del día 24, lo que permite apreciar que no hubo una relación directa entre el aborto y lo avanzado en la maduración del endometrio. Antes por el contrario, creemos que el procedimiento mejorará las condiciones endometriales que facilita la implantación ovular.

\section{SUMMARY}

We are presenting a report of pregnancies which occur after an endometrial biopsy. All theese patients were seen because of sterility.

The endocrinological evaluation was done by specific laboratory tests in blood and endometrial histology.

25 pregnancies $(11.1 \%)$, out of 223 , occured after a biopsy was done and are analized in this paper.

\section{RESUMEN}

Se presenta un informe de los embarazos obtenidos en el ciclo durante el cual se practicó la biopsia de endometrio, en pacientes que consultatron por esterilidad. El estudio anatomopatológico del endometrio junto con el estudio de efectores y las determinaciones hormonales específicas, son exámenes que permiten una adecuada evaluación del factor endocrino.

Se analizaron 25 pacientes (11.1\%) que se embarazaron durante el ciclo de la biopsia, del total de 223 embarazos de pacientes estériles. Tres de los embarazos se lograron durante el período de tratamiento y el resto (22) durante la fase de estudio. Sólo 8 pacientes tenían diagnóstico etiológico de su esterilidad. El informe histológico del endometrio fue secretor en 23 y en dos, proliferativo. Catorce pacientes (56\%) tuvieron un embarazo a término y un $12 \%$ abortos. Se discute el posible mecanismo que desencadena la presencia de embarazos durante este procedimiento en pacientes estériles.

3 pregnancies were obtained during the treatment period and, the others (22), during the study phase.

The etiology of their sterility was found only in 8 patients. In 23 the endometrial histologycal report was secretor and proliferative in two. A normal pregnancy was obtained in 14 patients (56\%) and $12 \%$ (3 patients) of abortions was seen.

The mecanism of theese pregnancies after a endometrial biopsy in sterile patients is in discussion yet.

\section{BIBLIOGRAFIA}

1. WENTZ, A.C. Endometrial biopsy in the evaluation of infertility. Fertil. Steril. 33: 121, 1980.

2. DRIESSEN, F.; HOLWERDA, P.J.; PUTTE, S.C.J.; KREMER, $J$. The significance of dating and endometrial biopsy for the prognosis of the infertile couple. Int. J. Fertil. 25: 112, 1980.
3. NOYES, R.W.; HERTING, A.T.; ROCK, J. Dating the endometrial biopsy. Fertil. Steril. 1: 3, 1950.

4. VAN BOGAERT, L.J.; MALDOGNe, P.; STAQUET, J.P. Endometrial biopsy interpretation. Shortcomings and problems in current gynecologic practice. Obstet. Gynecol. 51: 25,1978 . 
5. MISHELL, D.R.; MOTLYOFF. Practical value of endometrial biopsies. N.Y. State J. Med. 40: 928, 1940.

6. STURGES, S.H. Diagnostic significance of endometrial glands in early pregnancy. Am. J. Obstet. Gynecol. 39: 10, 1940.

7. WILSON, R.B.; LEE, R.A.; JENSEN, P.A. Inadvertent infertility investigation in pregnant women. Fertil. Steril. 17: 126, 1966.

8. BUXTON, C.L., OLSON, L.E. Endometrial biopsy inadvertently taken during concepcion cycle. Am. J. Obstet. Gynecol. 105: 702, 1969.

9. KAROW, W.G., GENTRY, W.C., SKEELS, R.F.; PAYNE, S.A. Endometrial biopsy in the luteal phase of the cycle of conception. Fertil. Steril 22: 482, 1971.

10. ROSENFELD, D.L.; GARCIA, C.R. Endometrial biopsy in the cycle of conception. Fertil. Steril. 26: 1088, 1975.

11. SULEWSKI, J.M.; WARD, S.P.; Mc GAFFIC, W. Endometrial biopsy during a cycle of conception. Fertil. Steril. 34: 548, 1980.

12. SANCHEZ, F.; DEVIA, G.; HENAO, G.; DIAZ, C.; MEJIA, J.M. Embarazos en 223 pacientes tratados por esterilidad durante un período de cinco años. Rev. Col. Obstet. Ginecol. 33: $183,1982$.

13. INDUÑATE, D.M.; CORREA, C.C.; ARREOLA, M.A.; LOPEZ, J.J.; HERNANDEZ - AYUP, S. Amenorrea primaria de etiología fímica. Ginecol. Obstet. Méx. 46: 363, 1979.
14. PILONIETA, C.J.; GARCIA, J. y Cols. Estudio de la Amenorrea Primaria durante el período de cuatro años (1976 - 1980). Rev. Colomb. Obstet. Ginecol. 32: 362, 1981.

15. TOAFF, R.; BALLAS, S. Traumatic hypomenorrhea Amenorrhea (Asherman's Syndrome) in Modern Trends in infertility and conception control. Edits. Wilkins Edward, E. Wallach and Kempers R.D. Williams Wilkins Edward, E. Wallach and Kempers, R.D. Williams Wilkins - Baltimore 1979 pag. 66.

16. ROCK, J.A. ZACUR, H.A. The clinical management of repeated early pregnancy Wastage. Fertil. Steril. 39: 123, 1983.

17. MOGHISSI, K.S.; WALLACH, E.E. Unexplained Infertility. Fertil. Steril. 39: 5, 1983.

18. SANCHEZ, F.; JACOME, E.; MORAN, C. y Col. Modificaciones endocrinas al estímulo vaginal y cervical. Rev. Latinoam. Fertil. Estéril. (En prensa).

19. VILAR-ROJAS, C.; HICKS, J.J. Prostaglandinas y reproducción: ovulación, transporte e implantación. Ginecol. Obstet. Mex. 50: 15, 1982.

20. BRONSON, R.; HAMADA, Y. The effect of protaglandins $\mathbf{F}_{2} \alpha$ and $\mathbf{E}$ on pregnancy in mice during implantation. Fertil. Steril. 30: 354, 1978.

21. MASLER, I.A.; RIDDICK, D.H. Prolactin production by human endometrium during the normal menstrual cycle. Am. J. Obstet. Gynecol. 135: 751, 1979. 